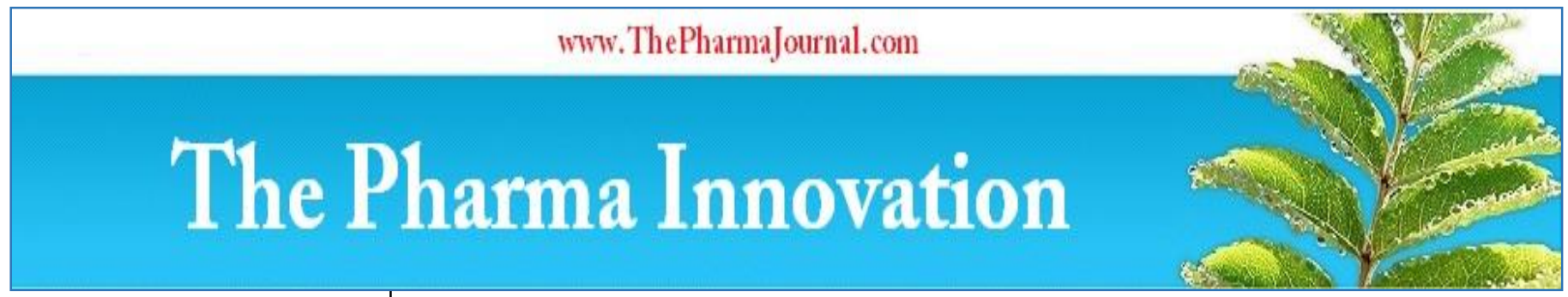

ISSN (E): 2277 - 7695

ISSN (P): 2349-8242

NAAS Rating: $\mathbf{5 . 0 3}$

TPI 2021; 10(3): 259-260

(C) $2021 \mathrm{TPI}$

www.thepharmajournal.com

Received: 14-01-2021

Accepted: 22-02-2021

Anugya Bharti

Department of Foods and

Nutrition, GB Pant University

of Agriculture and Technology,

Pantnagar, Uttarakhand, India

Kalpana Kulshrestha

Department of Foods and

Nutrition, GB Pant University

of Agriculture and Technology,

Pantnagar, Uttarakhand, India

Archana Kushwaha

Department of Foods and

Nutrition, GB Pant University

of Agriculture and Technology,

Pantnagar, Uttarakhand, India
Corresponding Author: Anugya Bharti

Department of Foods and

Nutrition, GB Pant University of Agriculture and Technology,

Pantnagar, Uttarakhand, India

\section{Enhancing nutrition knowledge: A step towards childhood obesity prevention}

\author{
Anugya Bharti, Kalpana Kulshrestha and Archana Kushwaha
}

DOI: $\underline{\text { https://doi.org/10.22271/tpi.2021.v10.i3d.5785 }}$

\section{Abstract}

The prevalence of overweight and obesity has increased substantially over the past three decades. Childhood obesity is a serious public health concern. Changes in the dietary pattern and sedentary lifestyle contribute towards childhood obesity. Obese children grow into obese adult. Effective strategies must be taken for its prevention. Enhancing nutrition knowledge by means of nutrition education contributes towards healthier lifestyle choice that includes proper diet management and physical activity and thus helps to prevent childhood obesity to a great extent. In the present study, nutrition knowledge was assessed and then two exposures were given by means of nutrition education video. Result of the study shows that after repeated exposure, there was gain in knowledge among the children.

Keywords: Enhancing, nutrition, towards, childhood, prevention

\section{Introduction}

Obesity is defined as condition of excessive fat accumulation in adipose tissue, to an extent that health may be impaired (www.who.int). Childhood obesity is increasing at alarming rate every year all over the world and responsible for causing various health problems such as diabetes, hypertension, heart disease and various other psychological problems (Raj and Kumar, 2010) ${ }^{[1]}$. Faulty eating habits, changes in the dietary pattern and sedentary lifestyle contribute towards childhood obesity. Increasing prevalence of childhood obesity is a serious matter of concern as overweight and obese children grow into obese adult, thus, increases medical cost and add burden to society. So, steps should be taken to determine the prevalence. But only assessing the obese child is not solution to prevent obesity. Prevention is better than cure. So, along with identification of obese child, it is important to change the root cause of obesity as healthy children only builds healthy communities. Children with limited nutrition knowledge and unhealthy eating habits were five times more likely to be obese than those children having nutrition knowledge according to Trichesa and Giuglianib (2005) ${ }^{\text {[2] }}$. So, Verrotti et al. (2014) ${ }^{[3]}$ stated that changing the lifestyle in childhood toward healthy eating habits and physical activity through educational interventions at school level can play important role in the prevention of obesity as it is seen that it is difficult to treat obesity in adults through changes in their lifestyle rather than during childhood. This can be done by imparting nutrition knowledge to children regarding nutritious and healthy food, importance of physical activity, etc. so that they can adopt healthier lifestyle choices than they had prior to intervention. Health education, nutrition education, proper diet management and physical activity help to prevent childhood obesity to a great extent.

Keeping in view the above points, the present study was planned with objective that include development of module to impart nutrition education and generating nutrition awareness among overweight and obese children and to see its impact on knowledge gain for reducing the incidence of childhood obesity.

\section{Materials and Methods}

A study was conducted in schools of G.B. Pant University of Agriculture and Technology, Pantnagar Campus, U.S. Nagar (Uttarakhand) among 36 overweight/obese children studying in class 4 to $5^{\text {th }}$ standard. Samples were selected by multistage purposive sampling. Permission was taken from Principal and informed consent was obtained from the child also before conducting the study. The anthropometric measurements were recorded for all the subjects and their health status was determined. In order to impart nutrition education to students, first their nutritional knowledge was assessed. 
Ten questions were framed and were asked from subjects and their response was recorded according to response scale that includes right, wrong and no answer. For every right answer, one mark was awarded and for wrong or no answer no marks was given. Children giving right answer for 1 to 3 questions were categorized under poor category, children giving right answer for 4 to 6 questions were categorized under fair category and children nutrition knowledge was considered as good, if he/ she gave right answer for more than 6 questions. In this way, children knowledge about nutrition was assessed before imparting nutrition education. After pre - testing of their nutrition knowledge, nutrition education video was shown to these children and again unanswered/ wrong questions were asked and their response was recorded. Children who were able to answer all questions at this stage were send to their respective class and video was again shown to rest of them. Again unanswered/ wrong questions were asked from rest children and their response was recorded again. Thus, pre - testing and two post- testing of nutrition knowledge was done. Data was analysed in SPSS version 20 and $p<0.05$ were considered as statistically significant. Paired $\mathrm{t}$ - test was applied to see the impact of nutrition education on knowledge gain of children.

\section{Development of module for nutrition education}

Module named "Eat healthy, remain active and prevent Childhood Obesity" was developed for imparting nutrition education and generating nutrition awareness among overweight and obese children. Module was developed in video form as children were smaller in age ( $6-12$ years) and education in video form would have been attractive and could be easily understood by them. Module includes information under following heads:

- What is food and functions of food in body

- Types of food groups that we eat. It includes carbohydrates, proteins, fats, vitamins and minerals. Also, sources and function of these food groups in the body.

- Functions of water in body

- Food pyramid showing minimum serving of cereals, pulses, fruits and vegetables, meat and poultry, milk and milk products and fats and oils to be included in diet for healthy body.

- Childhood obesity occurring due to energy imbalance i.e. when energy intake is more and energy expenditure is less.

- Importance of physical activity in reducing childhood obesity

- Avoid consumption of fast foods such as maggi, macroni, pizza, chowmin, samosa, etc.

- Foods to be avoided and to be included in diet for good health

\section{Results and Discussion}

Results of pre-exposure and two post - exposure (post exposure 1 and post - exposure 2) are shown in Table1. Results of the study shows that in pre - exposure, only $19.44 \%$ children were having good nutrition knowledge whereas rest were having fair or poor knowledge. Knowledge of children was enhanced after repeated exposure. After two exposures, all children were falling in good nutrition knowledge category. Although, it was seen that not all 36 students were able to give right answer for all 10 questions but were able to answer for at least more than 6 questions.
After repeated exposure, children with poor nutrition knowledge decreases and that with good knowledge increases when exposed to nutrition education video. Result of paired $t$ -test shows significant association between pre-exposure and post - exposure 1; between post - exposure 1 and post exposure 2 at 0.05 level of significance with $p$-value less than $0.05(p<0.05)$. This shows that there was gain in knowledge after children were exposed to nutrition education.

Table 1: Knowledge status of children before and after exposure

\begin{tabular}{|c|c|c|c|c|c|c|}
\hline & \multicolumn{2}{|c|}{ Poor } & \multicolumn{2}{c|}{ Fair } & \multicolumn{2}{c|}{ Good } \\
\hline & N & \% & N & \% & N & \% \\
\hline Pre - exposure & 5 & 13.89 & 24 & 66.67 & 7 & 19.44 \\
\hline Post - exposure 1 & 1 & 2.79 & 12 & 33.33 & 23 & 63.89 \\
\hline Post - exposure 2 & 0 & 0.00 & 0 & 0.00 & 36 & 100.00 \\
\hline
\end{tabular}

\section{Conclusion}

Childhood obesity is major public health problem. Prevalence of childhood obesity is increasing worldwide and is responsible for various diseases. Factors responsible for this must be assessed and effective strategies must be planned for prevention. Creating awareness among children by imparting nutrition education is helpful in preventing childhood obesity to a large extent.

\section{References}

1. Raj Manu, Kumar Krishna R. Obesity in children \& adolescents. Indian Journal of Medical Research 2010;132:598-607.

2. Trichesa RM, Giuglianib ERJ. Obesity, eating habits and nutritional knowledge among school children. Rev Saude Publica 2005;39(4):P1.

3. Verrotti A, Penta L, Zenzeri L, Agostinelli S, Feo PD. Childhood obesity: prevention and strategies of intervention. A systematic review of school-based interventions in primary schools. Journal of Endocrinological Investigation 2014;37(12):1155-1164. www.who.int/mediacentre/factsheets/fs311/en/

4. www.who.int/topics/obesity/en/. Health topics-Obesity 